\title{
Aplicativo en la evaluación y acreditación de universidades con carreras técnicas
}

Application in the evaluation and accreditation of universities with technical degrees Aplicação na avaliação e acreditação de universidades com formação técnica

\section{Renso Carrasco L.}

Dr.C, Universidad de Cartagena, Cartagena, Bogot, https://orcid.org/0000-0002-2544-8392, rensocarrasco@gmail.com

\footnotetext{
Recibido 4 octubre 2019 - Aceptado 12 mayo 2020

Formación docente - revista iberoamericana de educación

http://www.revista-iberoamericana.org/index.php/es/index https://creativecommons.org/licenses/by/4.0/deed.es e-ISSN: 2737-632X
}

Vol - 3 No. 3, julio - septiembre 2020

Pags 53 - 68

Resumen Higher Education in Ecuador entered a stage of changes driven by the regulations that govern the system as of 2008, such situation has required universities and polytechnical schools to start continuous improvement processes based on periodic self-assessments taking as reference different models Of quality and in particular that issued by the Council of Evaluation, Accreditation and Quality Assurance, CEAACES and whose purpose is to accredit as well as their careers. The establishment of the evidence that justifies the quality indicator, as well as the weight given to each is the substantial part of the selfevaluation process that the institutions carry out and it is necessary to have a tool that minimizes subjectivity and generates Results that in principle can be good but that in the end do not reach for the external evaluation; For which it has been made a tour of different concepts, regulations and processes that are applied in Ecuador for the evaluation of careers based on a generic model establishing a procedure that has been used to determine the weights of the indicators and proposes some lines Of future work. 
Palabras clave: Career Assessment, criteria, quality indicators, self appraisal

Abstract. Higher Education in Ecuador entered a stage of changes driven by the regulations that govern the system as of 2008, such situation has required universities and polytechnical schools to start continuous improvement processes based on periodic self-assessments taking as reference different models Of quality and in particular that issued by the Council of Evaluation, Accreditation and Quality Assurance, CEAACES and whose purpose is to accredit as well as their careers. The establishment of the evidence that justifies the quality indicator, as well as the weight given to each is the substantial part of the selfevaluation process that the institutions carry out and it is necessary to have a tool that minimizes subjectivity and generates Results that in principle can be good but that in the end do not reach for the external evaluation; For which it has been made a tour of different concepts, regulations and processes that are applied in Ecuador for the evaluation of careers based on a generic model establishing a procedure that has been used to determine the weights of the indicators and proposes some lines Of future work.

Key words: Career Assessment, criteria, quality indicators, self appraisal

Resumo O ensino superior no Equador entrou em um estágio de mudanças impulsionadas pelos regulamentos que governam o sistema a partir de 2008. Tal situação exigiu que as universidades e as escolas politécnicas iniciassem processos de melhoria contínua com base em autoavaliações periódicas, tomando como referência modelos diferentes de qualidade e, especialmente, aquele que foi emitido pelo Conselho de Avaliação, Acreditação e Garantia da Qualidade, CEAACES e que se destinam a provar a si mesmos e a suas carreiras. O estabelecimento de evidências que justifiquem o indicador de qualidade, bem como o peso atribuído a cada um, é parte substancial do processo de 
autoavaliação que as instituições realizam e é necessário ter uma ferramenta que minimize a subjetividade e gere resultados que, em princípio, podem ser bons, mas que, no final, não são suficientes para a avaliação externa; razão pela qual um passeio foi realizado por meio de diferentes conceitos, regulamentos e processos aplicados no Equador para a avaliação de carreiras com base em um modelo genérico, estabelecendo um procedimento que foi usado para determinar os pesos dos indicadores e propõe algumas linhas de trabalhos futuros.

Palavras-chave: Avaliação de carreira, critérios, indicadores de qualidade, autoavaliação.

\section{INTRODUCCIÓN}

Tomar una decisión involucra considerar varios criterios y posiblemente estos bajo incertidumbre, la teoría de la decisión nos ayuda con metodologías y herramientas para tratar de dar solución a problemas complejos.

Estos problemas complejos se presentan en la vida cotidiana prácticamente en todas las actividades del ser humano por lo que las consecuencias de estas decisiones se ven reflejadas a corto, mediano o largo plazo según sea el caso. Se requiere entonces un enfoque objetivo hacia la toma de las decisiones basada en el análisis de datos y la información, esto a su vez constituye uno de los aspectos básicos en un sistema de gestión de la calidad y mejora continua.

Desde este punto de vista y en el ámbito de la educación a partir del año 1990 en Latinoamérica comienzan los primeros esfuerzos entorno a la evaluación de los procesos de enseñanza aprendizaje con miras a asegurar niveles de calidad y excelencia para lo cual los planteamientos en la mejora continua juegan un papel importante.

El Ecuador también entra a formar parte de esta tendencia a partir del año 2007 con procesos de evaluación y acreditación de las universidades que eran parte de su sistema de educación superior. 
Para el año 2010 la Asamblea Nacional del Ecuador como órgano legislativo expide la Ley Orgánica de Educación Superior, LOES, que en uno de sus artículos crea los organismos públicos que rigen el Sistema de Educación Superior, entre ellos al CEAACES organismo que dentro de sus funciones constan la planificación, coordinación y ejecución de los procesos de evaluación, acreditación, clasificación, aseguramiento de la calidad, aprobación de la normativa para estos procesos así como el establecimiento de los criterios e indicadores de calidad como también de los instrumentos a aplicarse.

La Universidad Estatal Península de Santa Elena, UPSE, es una institución de educación superior creada el 22 de julio de 1998 con Registro Oficial No. 366, que ha experimentado tres evaluaciones institucionales y a la fecha se encuentra acreditada en la categoría C hasta el año 2018, sin embargo tal como lo establece la LOES, las instituciones de educación superior deben ser evaluadas y acreditadas así como sus carreras y programas.

El aseguramiento de la calidad en la educación superior se relaciona con una diversidad de propósitos y marcos metodológicos, que reflejan distintos intereses y necesidades, y, por supuesto, al uso que se dará a los resultados. En general, estos propósitos pueden agruparse en tres grandes categorías, que no son mutuamente excluyentes sino que expresan énfasis dominantes en contextos específicos. Estos son:

El Control de Calidad, expresado en procesos de licenciamiento o autorización de funcionamiento. El control de calidad se identifica con el rol tradicional del Estado de asegurar que la provisión educativa se ajusta a niveles mínimos de calidad. Cuanto los sistemas eran mayoritariamente públicos, esta función se desarrollaba de manera automática, basada en el supuesto (habitualmente correcto) de que la calidad era un rasgo propio de las instituciones públicas.

Cuando los sistemas se diversificaron, y la educación superior quedó en manos de una variedad de proveedores, se hizo indispensable desarrollar mecanismos 
que permitieran asegurar la operación de instituciones o programas que satisfacían estándares mínimos de calidad.

Así, ante el crecimiento en el número y/o diversidad de las instituciones de educación superior, varios países han desarrollado mecanismos que garantizan que sólo se autorizará la instalación y operación de instituciones o programas que satisfacen ciertos requisitos básicos de calidad. Estos mecanismos llevan distintas denominaciones, pero en la práctica corresponden a lo que puede llamarse licenciamiento o autorización, para indicar que otorgan una licencia básica para la operación como instituciones de educación superior, sobre la base de la verificación de las condiciones en que desarrollan su labor.

La garantía de la calidad, expresado en procesos de acreditación, de carreras o instituciones Aunque cuando se haya logrado asegurar un nivel básico de calidad, subsiste la necesidad de informar a los distintos actores acerca de la calidad de las ofertas educativas. Es necesario, además, dar cuenta pública de los recursos recibidos, ya sea del estado, de los estudiantes o de otras fuentes.

En este caso, el objetivo principal es la provisión de información confiable acerca del grado en que las instituciones o sus programas cumplen con lo que ofrecen y satisfacen las expectativas asociadas a un determinado grupo de referencia, sea éste disciplinario, profesional o laboral. Los sistemas de acreditación evalúan la institución, programa o carrera con relación a sus propios procesos y a un conjunto de estándares, y como consecuencia, dan garantía pública de su calidad, entendida ésta como la capacidad para cumplir tanto con los requerimientos internos (propósitos) como externos (estándares).

El Mejoramiento, expresado en procesos de auditoría académica. Este es el objetivo final de todo sistema de aseguramiento de la calidad, aun cuando en general se reconoce que las etapas previas son necesarias. El objetivo de mejoramiento enfatiza el hecho de que la responsabilidad por la calidad 
corresponde a las instituciones; por tanto, se centra en su capacidad para desarrollar y aplicar políticas y mecanismos eficaces de autorregulación y mejora continua. El procedimiento comúnmente asociado con la mejora es la auditoría académica, en que la evaluación se refiere a los propósitos institucionales y a la forma en que la institución vela por su logro oportuno, y es capaz de hacer los ajustes necesarios cuando detecta debilidades o áreas deficitarias.

América Latina está entre las regiones pioneras en el campo del aseguramiento de la calidad, considerando que con excepción de Estados Unidos, se trata de procesos relativamente jóvenes. En efecto, ya durante la década de los noventa, al menos Chile, Colombia y Argentina contaban con procesos en diversas etapas de consolidación. En Europa, en cambio, si bien en esos años existían procesos de evaluación, la acreditación comenzó a desarrollarse después del 2000, el mismo período en que comenzaron a desarrollarse procesos en Australia y varios países del Asia.

\section{MATERIALES Y MÉTODOS}

El modelo busca tomar en cuenta de forma integral todos los aspectos involucrados en la actividad universitaria por esta razón se basa en la metodología multicriterio.

El CEAACES justificó la utilización de esta metodología basada en:

- Investigación e integración de intereses y objetivos de múltiples actores.

- Complejidad de la configuración de los múltiples actores.

- Método de alternativas de valoración.

- Objetividad e inclusión de diferentes intereses de los actores.

Para el desarrollo del modelo se siguió la siguiente metodología:

- Identificación de los criterios principales e indicadores a ser medidos. 
- Diseño de guías de verificación in situ

- Aplicación de plataforma informática para el ingreso de información

- Diseño de las funciones de utilidad

- Definición de indicadores con mayor relevancia

- Asignación de pesos relativos por criterios, subcriterios e indicadores

- Análisis de funciones de utilidad

- Suma ponderada entre las utilidades de cada indicador para la valoración de los subcriterios.

Esta metodología sirve para tratar problemas de toma de decisiones en los que se presentan "diversos objetivos o criterios que simultáneamente deben incorporarse". (Ávila, 2000).

A partir de lo mencionado y para la aplicación de la metodología multicriterio se ha establecido los siguientes criterios: Pertinencia, Plan Curricular, Academia, Ambiente Institucional y Estudiantes.

Criterio Pertinencia: evalúa que la carrera o programa académico, responda a las expectativas y necesidades de la sociedad, a la planificación nacional, a la prospectiva de desarrollo científico, humanístico y tecnológico mundial y a la diversidad cultural.

Criterio Plan Curricular: evalúa los distintos niveles de concreción del currículo asegurando coherencia e integración entre el macro, meso y microcurrículo.

Criterio Academia: evalúa a los profesores de la carrera. Los profesores son el personal encargado directamente del proceso de enseñanza aprendizaje, de la investigación y la vinculación, quienes deben tener las competencias necesarias para cumplir todas las áreas curriculares de la carrera. 
Criterio Ambiente Institucional: evalúa el sistema de gestión académica de la carrera ejecutado por una coordinación / dirección responsable de los procesos de seguimiento académico curricular y de recursos de apoyo.

Criterio Estudiantes: evalúa la participación estudiantil en actividades complementarias, tutorías, políticas de bienestar estudiantil y el proceso de acreditación de las carreras.

La metodología utilizada para la evaluación de carreras con la aplicación del modelo propuesto por el ente evaluador, propone una asignación de valoraciones de desempeño (utilidades) a cada uno de los indicadores del modelo; esta asignación se efectúa de acuerdo con ciertas funciones de utilidad. Para la aplicación de lo mencionado en el caso de los indicadores cualitativos, se tiene:

- 1 para el nivel satisfactorio,

- 0,5 para medianamente satisfactorio y

- 0 para deficiente.

- En el caso de los indicadores cuantitativos y mixtos, se tiene una función matemática que adjudica un valor de desempeño entre 0 y 1 .

Para obtener un resultado final de la carrera en el entorno de aprendizaje se utiliza una suma ponderada de los desempeños de todos los indicadores.

La determinación de las variables es la primera acción que se toma puesto que en base a estas variables se identifican los indicadores que son parte del modelo. Estas variables son calculadas a partir de los datos que se obtienen directamente de las carreras.

Para el caso de los indicadores cualitativos la variable y el indicador coinciden. 
El modelo de evaluación del entorno de aprendizaje de la carrera utilizado para nuestra aplicación tiene un total de 37 indicadores de los cuales 17 son cuantitativos y 20 cualitativos.

La UPSE, dispone de una dependencia de asesoría y apoyo para procesos de aseguramiento de calidad, posee la Unidad Operativa de Acreditación, desde esta oficina se dicta lineamientos que permitan el cumplimiento de estándares de calidad, así como también se sugieren y en algunos casos se disponen los pasos a seguir como institución.

Los pesos que se dé a cada criterio, subcriterio e indicador van a ser el factor diferenciador que potencia a una institución de otra dependiendo siempre de factores sociales, regionales, económicos, etc., que están asociados a la institución. Existen tres comisiones especializadas que aportan para los temas de calidad, estas son:

- Comisión de Evaluación, Acreditación y Aseguramiento de la calidad.

- Unidad Operativa de Acreditación.

- Comité o Sub-comisión de Evaluación Interna de las Carreras.

La Comisión de Evaluación, Acreditación y Aseguramiento de la Calidad es la responsable de la ejecución de los procesos de autoevaluación, así como del seguimiento de las políticas y planes que implementen las instituciones para la mejora continua. Estará conformado por representantes de autoridad, profesores, estudiantes, personal administrativo.

La Unidad Operativa de Acreditación es la responsable de ejecutar las políticas y decisiones de la comisión de evaluación, acreditación y aseguramiento de la calidad, aprueba en primera instancia los planes, políticas y acciones relativas a los procesos de autoevaluación. 
El Comité o Sub-comisión de Evaluación Interna de las Carreras tiene como función apoyar en forma permanente al Director de la Carrera en la planificación, ejecución y control del proceso de evaluación de carreras. Está integrada por el Director de la carrera, representante de docentes, representante de estudiantes, Técnico Docente y Asistente Administrativo.

El proceso de ponderación del modelo implicó varias fases, entre estas tenemos:

La conformación de comisiones, con la participación de docentes, estudiantes, profesionales y personal administrativo quienes son un factor importante con el propósito de disminuir el nivel de subjetividad de las ponderaciones, cada uno desde su perspectiva contribuye a disponer de una herramienta consistente para generar la utilidad final al aplicar el modelo.

Se establecieron cinco grupos, considerando que el modelo de trabajo tiene 5 grandes criterios; cada grupo está integrado por:Yo

- 3 Docentes a tiempo completo

- 1 estudiantes

- 1 Profesional externo

- 1 Asistente administrativo.

La Socialización del modelo de evaluación, en sesiones presenciales con todos los miembros de las comisiones, donde la idea principal está centrada en discutir los conceptos claves del aseguramiento de la calidad, evaluaciones históricas al sistema de educación superior, evaluación institucional y la estructura del modelo.

Cada uno de los indicadores puede ser evaluado de forma cuantitativa o cualitativa, el aporte que cada uno da dentro de su grupo y el punto de vista del modelo en relación a la situación institucional son temas que ameritan un gran debate. 
Actualmente en el Ecuador se dispone de varios documentos que aportan al análisis, como por ejemplo: el Modelo de Evaluación Institucional 2013, la Ley de Educación Superior, el Reglamento de Régimen Académico, el Reglamento de Escalafón del Docente e Investigador, el Modelo de Evaluación de las Carreras de Medicina y demás normativa interna que guía el accionar y cumplimiento de la misión institucional.

Talleres de Ponderación, que define tres etapas para la actividad:

- Esquema de Ponderación.

- Ponderación de criterios.

- Ponderación de Subcriterios e Indicadores

- Para el esquema de ponderación se tiene:

- La sumatoria total de las ponderaciones será igual a 100 puntos.

- Cada criterio tendrá un valor que representará el 100\% en su nivel.

- La suma de las utilidades de los indicadores y subcriterios deberá ser igual a la suma del grupo contenedor que corresponda.

- Para la valoración final se utilizará las funciones de valor y las funciones de utilidad dependiendo de la forma de medición de cada indicador.

La ponderación de criterios, considera varios aspectos que tiene relación con la realidad de la institución, el contexto del sistema de educación y la perspectiva de la carrera, representado en los siguientes enunciados:

- Situación de la Oferta académica de las carreras según la nueva nomenclatura de títulos.

- La oferta académica vigente y la necesidad de garantizar un buen proceso de formación.

- El nivel académico y rol del docente.

- La producción científica. 
- Los procesos institucionales.

- El proceso de formación expresado en profesionales que termina la carrera.

La Ponderación de Subcriterios e Indicadores, sigue la misma metodología aplicada para los criterios, además, la suma de los subcriterios dará como resultado la utilidad del criterio, así como la suma de los indicadores será igual a la utilidad del subcriterio correspondiente.

\section{RESULTADOS}

El modelo genérico que propone el Consejo de Evaluación, Acreditación y Aseguramiento de la calidad para la evaluación de carreras, contiene una serie de variables de carácter discrecional, que combinada a las variables cuantitativas generan una utilidad que al final del ejercicio genera un resultado cuyo peso está más ligado al criterio personal que al dato cuantificable. Se requiere demostrar cómo se puede minimizar el impacto de una evaluación basada en criterio del evaluador y utilizar otro mecanismo para dar valor a la variable.

El modelo en estudio contempla una serie de evidencias aceptables como cumplimiento del indicador en su respectiva variable, la discrecionalidad con que se juzga los indicadores cualitativos en comparación con lo exacto de los cuantitativos merece una reflexión de que es mejor sin que eso afecte la calidad.

La legislación del país determina que la acreditadora del Estado es el ente que certifica la calidad de los programas, carreras y la institución en su conjunto, sería muy importante desde el Sistema de Educación Superior, poder generar un modelo que permita valorar la calidad y aplicarlo como estrategia de mejora continua y que sus resultados permitan cumplir estándares no solo nacionales sino internacionales. 
Indudablemente que cada una de las acciones que se realicen deben tener un mayor o menor grado de aproximación al valor exacto, sin embargo, queda en el debate si el instrumento con el que se mide aporta, la forma como se asigna la utilidad es pertinente, los niveles de agrupamiento son consistentes. En el año 2010, luego de la evaluación que realizará el CONEA a las universidades hubo muchos cuestionamientos al modelo aplicado y de a poco se ha ido mejorando, sería importante valorar la eficacia del modelo y compararlo con otros similares en sus fines.

\section{CONCLUSIONES}

Comportamiento de variables cuantitativas y cualitativas que propone el Consejo de Evaluación, Acreditación y Aseguramiento de la Calidad para la evaluación de carreras, contiene una serie de variables de carácter discrecional, que combinada a las variables cuantitativas generan una utilidad que al final del ejercicio genera un resultado cuyo peso está más ligado al criterio personal que al dato cuantificable. Se requiere demostrar cómo se puede minimizar el impacto de una evaluación basada en criterio del evaluador y utilizar otro mecanismo para dar valor a la variable.

El modelo en estudio contempla una serie de evidencias aceptables como cumplimiento del indicador en su respectiva variable, la discrecionalidad con que se juzga los indicadores cualitativos en comparación con lo exacto de los cuantitativos merece una reflexión de que es mejor sin que eso afecte la calidad.

Modelo de evaluación para carreras en la legislación del país determina que la acreditadora del Estado es el ente que certifica la calidad de los programas, carreras y la institución en su conjunto, sería muy importante desde el Sistema de Educación Superior, poder generar un modelo que permita valorar la 
calidad y aplicarlo como estrategia de mejora continua y que sus resultados permitan cumplir estándares no solo nacionales sino internacionales.

Los algoritmos de medición de calidad indudablemente que cada una de las acciones que se realicen deben tener un mayor o menor grado de aproximación al valor exacto, sin embargo, queda en el debate si el instrumento con el que se mide aporta, la forma como se asigna la utilidad es pertinente, los niveles de agrupamiento son consistentes. En el año 2010, luego de la evaluación que realizará el CONEA a las universidades hubo muchos cuestionamientos al modelo aplicado y de a poco se ha ido mejorando, sería importante valorar la eficacia del modelo y compararlo con otros similares en sus fines.

\section{REFERENCIAS}

Ávila, R. (2000). El AHP (Proceso Analítico Jerárquico) y su aplicación para determinar los usos de las tierras. Santiago de Chile.

Aznar Bellver, J. (2012). Nuevos Métodos de Valoración - Modelos Multicriterio. Valencia: Editorial Universitat Politécnica de Valencia.

Bárcena, A. (2013). Estudio Económico de América Latina y el Caribe. Santiago de Chile: Naciones Unidas.

Barros-Bastidas, C., \& Turpo, O. (2020). La formación en investigación y su incidencia en la producción científica del profesorado de educa- ción de una universidad pública de Ecuador. Publicaciones, 50(2), 167-185. doi:10.30827/publicaciones.v50i2.13952

Barros Bastidas, C., \& Turpo Gebera, O. (2018). Factors influencing the scientific production of university professors: a systematic review 
https://doi.org/10.21803/pensam.v11i21-1.276

Bernal, S., Martínez, M., Parra, A., \& Jiménez, J. (2015). Investigación Documental Sobre Calidad De La Educación En Instituciones Educativas del Contexto Iberoamericano. Entramados - Ecuación y Sociedad.

Botero, C. (2009). Cinco Tendencias de la Gestión Educativa. Revista Iberoamericana de Educación.

CEAACES. (2012). Modelo Genérico para la Evaluación de Universidades.

CEAACES. (2013). Informe General sobre la Evaluación, Acreditación y Categorización de las Universidades y Escuelas Politécnicas.

CEAACES. (2015). Modelo genérico de evaluación del entorno del aprendizaje de carreras presenciales y semipresenciales de las Universidades y Escuelas Politécnicas del Ecuador. Ecuador.

CES. (Noviembre de 2013). Reglamento Régimen Académico del Sistema Nacional de Educación Superior. San Francisco de Quito, Ecuador.

CINDA. (2007). Eduación Superior en Iberoamérica, Informe 2007. Santiago de Chile: RIL*Editores.

Ecuador. (2008). Constitución de la República del Ecuador. Registro Oficial No. 449.

Ecuador. (2010). Ley Orgánica de Eduación Superior. Registro Oficial No. 298.

García, E. G. (1999). Evaluación y Calidad de la Universidad: El proyecto de la Universidad Complutense de Madrid. Revista Electrónica Interuniversitaria de Formación del Profesorado. 
Lemus, Edel and von Feigenblatt, Otto Federico and Orta, Miguel and Rivero, Orlando, Starbucks Corporation: Leading Innovation in the 21st Century (April 8, 2015). Journal of Alternative Perspectives in the Social Sciences, $7(1), \quad 23-38, \quad 2015, \quad$ Available at SSRN: $\underline{\text { https://ssrn.com/abstract }=2638468}$

von Feigenblatt, Otto Federico, ASEAN and Human Security: Challenges and Opportunities (July 29, 2009). Ritsumeikan Center for Asia Pacific Studies Working Paper, No. 09-5, Available at SSRN: https://ssrn.com/abstract=1442476 or http://dx.doi.org/10.2139/s $\underline{\operatorname{srn} .1442476}$

von Feigenblatt, Otto Federico, Japanese Animation as a Global Product: The Lingering Traces of Nijonjinron and the Rise of Globalism and Hybridity (August 2, 2012). Journal of History \& Social Sciences, 2(2), pp. 1-14, July-December 2012 , Available at

SSRN: https://ssrn.com/abstract $=2195562$ 